\title{
Pelvic floor muscle displacement during voluntary and involuntary activation in continent and incontinent women: a systematic review
}

\author{
Monika Leitner ${ }^{1,2}$ • Helene Moser ${ }^{1}$ - Jan Taeymans ${ }^{1,4,5}$ - Annette Kuhn ${ }^{3}$. \\ Lorenz Radlinger ${ }^{1}$
}

Received: 17 December 2014 / Accepted: 17 March 2015 / Published online: 21 May 2015

(C) The International Urogynecological Association 2015

\begin{abstract}
Introduction Investigations of the dynamic function of female pelvic floor muscles (PFM) help us to understand the pathophysiology of stress urinary incontinence (SUI). Displacement measurements of PFM give insight into muscle activation and thus help to improve rehabilitation strategies. This systematic review (PROSPERO 2013: CRD42013006409) was performed to summarise the current evidence for PFM displacement during voluntary and involuntary activation in continent and incontinent women.

Methods MEDLINE, EMBASE, Cochrane and SPORTDiscus databases were searched using selected terminology reflecting the PICO approach. Screening of Google Scholar and congress abstracts added to further information. Original articles investigating PFM displacement were included if they reported on at least one of the aims of the review, e.g., method, test position, test activity, direction and quantification of displacement, as well as the comparison between continent and incontinent women. Titles and abstracts were screened by two reviewers.
\end{abstract}

Electronic supplementary material The online version of this article (doi:10.1007/s00192-015-2700-2) contains supplementary material, which is available to authorised users

Monika Leitner

monika.leitner@bfh.ch

1 Health Division/Physiotherapy, Bern University of Applied Sciences, Murtenstrasse 10, 3008 Bern, Switzerland

2 Graduate School for Health Sciences, University of Bern, Bern, Switzerland

3 Gynaecology, Bern University Hospital, Bern, Switzerland

4 Faculty of Sport and Rehabilitation Sciences, Vrije Universiteit Brussel, Brussels, Belgium

5 Faculty of Health Sciences, Antwerp University, Antwerp, Belgium
The papers included were reviewed by two individuals to ascertain whether they fulfilled the inclusion criteria and data were extracted on outcome parameters.

Results Forty-two predominantly observational studies fulfilled the inclusion criteria. A variety of measurement methods and calculations of displacement was presented. The sample was heterogeneous concerning age, parity and continence status. Test positions and test activities varied among the studies. Conclusions The findings summarise the present knowledge of PFM displacement, but still lack deeper comprehension of the SUI pathomechanism of involuntary, reflexive activation during functional activities. We therefore propose that future investigations focus on PFM dynamics during fast and stressful impact tasks.

Keywords Activities of daily living · Diagnostics · Stress urinary incontinence

\section{Introduction}

Pelvic floor muscle (PFM) displacement is associated with the pathophysiology of stress urinary incontinence (SUI). SUI lowers the quality of life and well-being [1-3] of affected women and reduces their participation in sports and fitness activities $[4,5]$. To guarantee continence the PFM needs to contract strongly [6-8], rapidly and reflexively [9-11]. The dynamic properties of the PFM, contractility and strength, can be evaluated with various diagnostic approaches such as palpation, electromyography, dynamometry, imaging procedures [12] and thereby increase the understanding of the SUI pathomechanism.

Up to now research into PFM function using ultrasound and MRI has been focused on voluntary contractions and on involuntary reflexive activation during coughing or straining manoeuvres. While the first studies demonstrated an anterior- 
cranial movement, the latter resulted in a downward displacement. However, it is unclear whether PFM displacement has been investigated during daily life activities provoking SUI that also include involuntary reflex activation (e.g. running, jumping, stair climbing). Also, the muscle action during functional activities has not yet been fully understood and thus the specific form of contraction and mechanism of change in pelvic floor rehabilitation remains unknown.

Muscle actions can be classified as isometric (no change in muscle length), concentric (shortening action) and eccentric (lengthening action) [13]. The pure form of these types of muscle actions is seldom found. Komi therefore suggests that the natural variation of muscle function is more often a stretch and shortening cycle (SSC) [14]. However, knowledge about this combination of eccentric and concentric actions in the PFM during impact load is still inadequate. The concentric and isometric muscle actions lead to a lift (elevation) and squeeze (constriction) of the pelvic floor [15-17]. Not much is known about the eccentric muscle action of the PFM and the displacement during functional impact load activities of daily life (e.g. stair-climbing, running).

Enhanced comprehension of the PFM kinematics and muscle action related to SUI is of utmost clinical relevance for the development of new and specific approaches in PFM training and SUI rehabilitation. The current literature shows that the dynamic function of PFM can be assessed directly as the displacement measurement of the muscles themselves or indirectly via the displacement of the bladder neck $(\mathrm{BN})$ or other urogenital structures that reflect PFM contraction/movement. To our knowledge no review has been undertaken to systematically compile an overview concerning this matter.

The PICO acronym was used to develop the review question (Table 1). The PICO framework is highly structured and known to be very good at identifying medical literature [18]. The five PICO components are:

1. The patient population or problem being addressed (P)

2. The interventions or exposure of interest (I)

3. The comparators $(\mathrm{C})$

4. The main outcome or endpoint of interest (O)

The aim of this systematic review was to present an overview of the current available evidence for PFM displacement during voluntary and reflex activation in women $(\mathrm{P})$, to evaluate assessment methods, test positions and test activities (I), to compare displacement in continent and incontinent women $(\mathrm{C})$, and to display the direction and quantification of displacement $(\mathrm{O})$.

\section{Materials and methods}

This systematic review was performed according to the Preferred Reporting Items for Systematic Reviews and Meta-
Analyses (PRISMA) guidelines [19]. Methods of the analysis and inclusion criteria were specified in advance and documented in a protocol that was reviewed and published by PROSPERO (www.crd.york.ac.uk/prospero).

\section{Eligibility criteria and search strategy}

Studies from January 1990 to December 2013 of female participants aged 19 to 64 years, without restriction in study design or language, were sought in the electronic databases MEDLINE, EMBASE, Cochrane and SPORTDiscus. The search strategy included displacement terms (e.g. displacement, descent, movement, muscle action, dynamics) in combination with measurement methods (e.g. ultrasound, MRI), test positions (e.g. supine, standing) and activities that have been performed (e.g. voluntary contraction, coughing). The full electronic search strategy is available online in the appendix (Electronic Supplementary Material 3-Table 5: search strategy). It has been peer-reviewed as part of the systematic review process by an experienced librarian as recommended in the guideline by Sampson et al. [20].

The last systematic search was run on 12 December 2013. Additional studies were sought in SPORTDiscus, a comprehensive, full-text database covering sports and sports medicine journals, and in Google Scholar. Furthermore, some papers were added by scanning reference lists of articles, consultation of experts in the field and screening abstracts presented at conferences from the International Continence Society 2005 to 2012 and from the World Confederation for Physical Therapy 2011. A limited updated literature search was performed from 12 December 2013 to 6 August 2014.

To assess eligibility, inclusion and exclusion criteria were determined in advance (Table 1). Two review authors (LM and $\mathrm{MH}$ ) independently screened the titles and abstracts of the detected studies for eligibility and, in case of disagreement, sought consensus through discussion. The same researchers independently screened full-text articles for inclusion or exclusion based on criteria that were determined in advance. Disagreements regarding the eligibility of particular studies were resolved through discussion and with a third reviewer (RL).

Studies have been included in spite of the existence of exclusion criteria such as prolapse [21-23], being male [24], anal incontinence [22] and urge incontinence $[25,26]$ if the data had been reported separately between the subject groups. In that case the data of only continent and SUI patients were extracted according to the a priori established criteria.

\section{Quality assessment and data extraction}

The methodological quality of the articles included was assessed using the Critical Appraisal Skills Programme (CASP; www.casp-uk.net) checklist for cohort studies. The criteria list 
Table 1 Inclusion and exclusion criteria for title, abstract and full-text evaluation

\begin{tabular}{lll}
\hline Item of PICO approach & Inclusion & Exclusion \\
\hline Population, problem & $\begin{array}{l}\text { Stress urinary incontinence, continence } \\
\text { Female, women } \\
\text { Pelvic floor, levator ani }\end{array}$ & $\begin{array}{c}\text { Faecal (anal) incontinence, cadaver, animals, diabetes, } \\
\text { neurological disease, prolapse, surgery (prolapse/incontinence), } \\
\text { urge urinary incontinence, male, pregnancy, adolescents, } \\
\text { bladder disease, drug therapy }\end{array}$ \\
Intervention & Ultrasound, MRI, motion tracking, visualisation \\
Comparison & Asymptomatic continent and incontinent women \\
Outcome & $\begin{array}{l}\text { Displacement, descent, pelvic floor muscle action, } \\
\text { bladder displacement, bladder neck descent, } \\
\text { urethrovesical positioning, angle of urethral }\end{array}$ \\
& inclination, ano-rectal angle \\
& Muscle function, contraction, kinematics & \\
\hline
\end{tabular}

(version 14.10.2010) comprised 12 items. Each item was scored with "+" if the criterion was fulfilled, with "_-" if the criterion was not fulfilled, and with "?" if the information was not provided or was unclear. All papers included were scored independently by two reviewers (LM and MH). Discrepancies were resolved by discussion or consensus. The results of the CASP checklist are summarised in Table 4 (available as electronic supplementary material 1).

Data extraction was performed independently by the two reviewers, using a customised data extraction sheet. The following data were extracted from the studies included: study design; characteristics of the subjects; evaluated method, test position and bladder filling; tested activity; direction and quantity of displacement; specifics of measurement; comparison of continent versus incontinent women; secondary outcome(s); conclusion. In the case of missing data, the corresponding author was contacted for clarification and any disagreement was solved in a consensus meeting with the other reviewers (RL and TJ).

\section{Data synthesis and analysis}

Because of the high degree of heterogeneity observed in the extracted data, the analysis was restricted to a systematic review and an a priori planned meta-analysis was not performed. Causes of heterogeneity are depicted in Table 2.

\section{Results}

\section{Study selection}

Figure 1 shows the flow chart of the search process. The search identified a total of 290 abstracts for inclusion in the review. The search of MEDLINE, EMBASE and Cochrane databases provided a total of 232 citations. Adjustment for duplicates was not needed. Fifty-eight records were additionally identified through the search in Google Scholar,
SPORTDiscus database, a hand search and conference abstracts (International Continence Society 2005 to 2012 and World Confederation for Physical Therapy 2011). Two hundred and six studies were discarded because they did not meet the inclusion criteria. Full texts of the remaining 84 articles were examined in more detail, of which 42 studies did not meet the inclusion criteria as described. One article was written in the French language and reviewed by HM and JT, who are proficient in that language. All the other studies were in English. Finally, 42 studies were included in the systematic review.

\section{Risk of bias within studies}

Table 4 (available as electronic supplementary material 1) displays the results of the methodological quality assessment. The studies included addressed a clear focused issue and used appropriate methods to answer their research questions. Recruitment was not clear in some studies owing to the abstract format that was accepted as an additional information source of the review. Exposure and outcome had been measured accurately and most of the times the item "follow-up" could not be answered. The result items 8 and 9 of the CASP checklist have been listed in the data extraction form.

The extracted data and major study characteristics are also shown in Table 4 (available as electronic supplementary material). Most studies had an observational study design.

\section{Measurement methods}

Measurement methods varied among the studies. Ultrasound imaging was used in 31 studies, with predominantly transperineal ultrasound (TPUS; 29), followed by transabdominal ultrasound (TAUS; 5), transvaginal ultrasound (TVUS; 1) and translabial ultrasound (TLUS; 1) techniques. Magnetic resonance imaging (MRI) was performed in 15 studies. There were three studies that compared two measurement methods in the same investigation [17, 25, 27]. Methods in 
Table 2 Causes of heterogeneity

\begin{tabular}{|c|c|}
\hline Clinical & Methodological \\
\hline $\begin{array}{l}\text { Recruitment of nulliparous versus } \\
\text { primiparous and multiparous women }\end{array}$ & $\begin{array}{l}\text { Measurement of different structures (bladder neck, bladder } \\
\text { base, bladder wall, urethra, ano-rectal angle) }\end{array}$ \\
\hline $\begin{array}{l}\text { Recruitment of continent versus } \\
\text { incontinent women }\end{array}$ & $\begin{array}{l}\text { Differences in specific measurement techniques (markers, } \\
\text { reference lines, coordinate systems) }\end{array}$ \\
\hline Various age groups & Differences in test positions \\
\hline \multirow[t]{4}{*}{ Difference in ethnic groups } & Differences in test activities \\
\hline & Differences in bladder filling \\
\hline & $\begin{array}{l}\text { Display of displacement in various units (metric }[\mathrm{mm}, \mathrm{cm}] \text {, } \\
\text { angle }\left[{ }^{\circ}\right] \text {, compliance }[\mathrm{C}] \text {, muscle strain }[\varepsilon] \text { ) }\end{array}$ \\
\hline & Differences in analysis: offline versus online \\
\hline
\end{tabular}

calculating the displacement varied substantially. Some TPUS studies that investigated the $\mathrm{BN}$ position referred to an $\mathrm{x}-\mathrm{y}$ coordination system originally described by Schaer et al. [28], whereas angles of $\mathrm{BN}$ rotation were analysed according to Balmforth et al. [29]. Besides metric units (mm, cm; 35 studies) and angular deviations (degrees; 8 studies) two studies also addressed the stiffness aspect in reporting muscle strain $(\varepsilon)$ [30] and compliance $\left(\mathrm{mm} / \mathrm{cmH}_{2} 0 ; \mathrm{BN}\right.$ mobility/ $\Delta$ rectal pressure) [31]. While the displacement of organs enables indirect observation, displacement of muscle structures allows direct conclusions of PFM function. The displaced structure that was assessed most was the BN (24 studies), followed by the bladder base ( 6 studies), the posterior and superior bladder wall
(3 studies), urethra, rectal ampulla, ano-rectal angle (ARA) and diaphragm. Five MRI and 2 TPUS investigations were able to directly measure the muscle displacement.

\section{Test positions and activities performed}

Most subjects had been tested in the supine position (21 studies), with a range of crook-lying and (semi)lithotomy position to semi-recumbent and half-sitting positions. There were two investigations in the sitting and 9 in standing position, whereas 2 studies did not specifically report the test position. Most researchers tested the PFM at rest, with voluntary PFM contraction (ranging from voluntary, gentle, moderate to
Fig. 1 Preferred Reporting Items for Systematic Reviews and Meta-Analyses (PRISMA) flow chart showing trial selection methodology

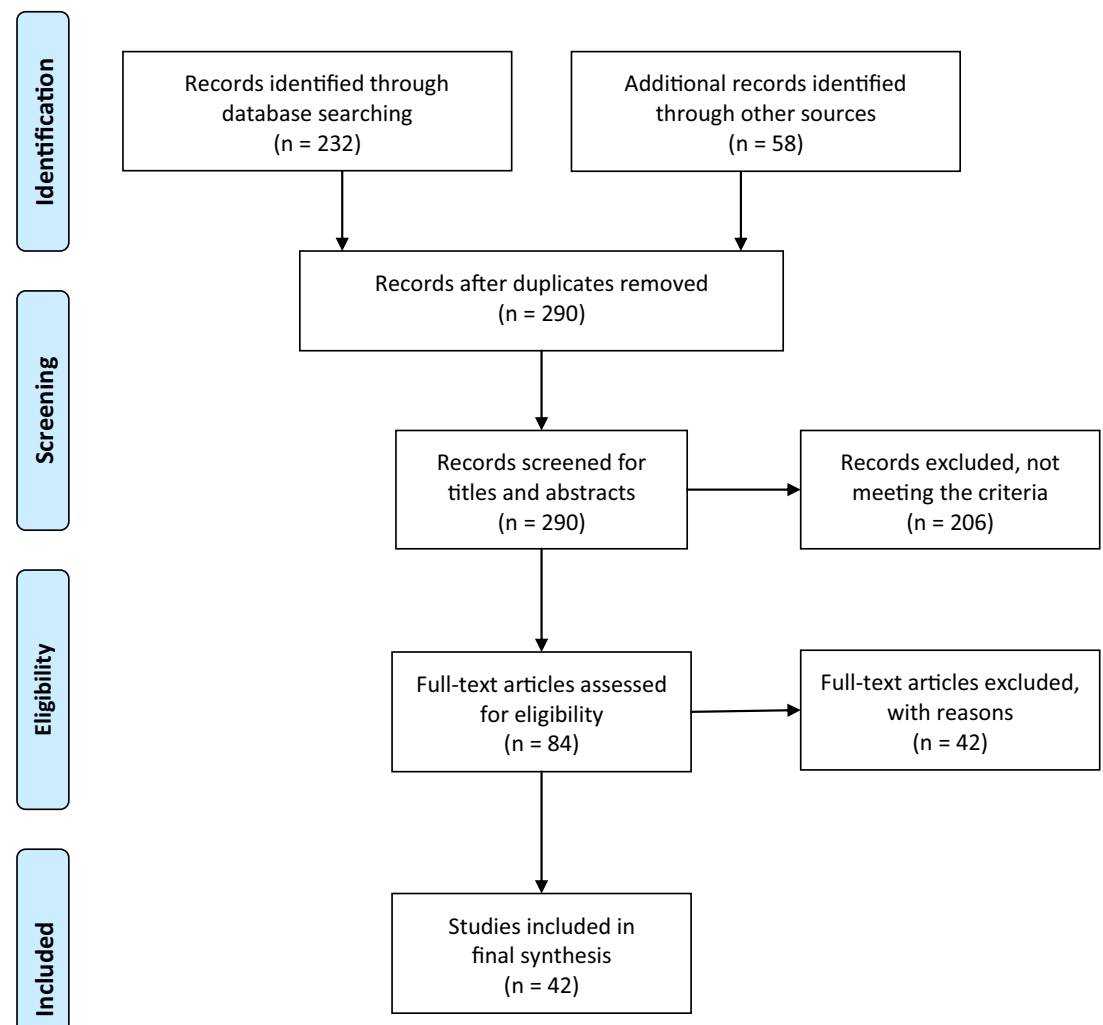


submaximal and maximal), Valsalva, straining and coughing. Also, breathing, abdominal curl-up, head lift, brace/ transversus abdominis muscle contraction and "The Knack" could be found as test activities. Predominantly the voluntary activation of the PFM had been assessed.

\section{Direction of the observed displacement}

There was broad consensus about the direction of PFM during voluntary contraction. It was described as lift, elevation in the cranial and ventral directions, posterior to anterior movement of the levator ani muscle (compressing the rectum towards the vagina and symphysis) or anterocephalic movement. Discrepancy was found in the Valsalva manoeuvre, with most authors describing descent, downward or dorso-caudal movement, whereas one study found a cranial motion (Fig. 2) [32]. Descent, ventral-dorsal/cranial-caudal, (dorso)-caudal direction were consistently found during coughing. One study confirmed the phase-locked parallel movement of the diaphragm and PFM during breathing by cranio-caudal movement of the diaphragm and pelvic floor in expiration and inspiration [33]. Wise et al. [34] showed that the BN moves in an arc of a circle, thus indicating the multi-directional character of $\mathrm{BN}$ displacement. Similar results followed in 2007 [35] and 2010 [36] with TPUS and motion tracking to analyse the dynamics of the ano-rectal angle, showing trajectories of movement and thus confirming the multi-directional displacement. Talasz et al. [33] described the respiratory-related parallel vertical movement of the diaphragm and pelvic floor.

\section{Quantification of the observed displacement}

In transabdominal ultrasound (TAUS) displacement measurements of the bladder base the lift component of voluntary PFM contraction ranged between $0.37 \mathrm{~cm} \mathrm{[37]} \mathrm{and} 0.87 \mathrm{~cm}$ [38] in the supine position. Kelly et al. [24] illustrated positional differences with $0.46 \mathrm{~cm}$ in the supine versus $0.69 \mathrm{~cm}$ in the standing position. Descent during Valsalva ranged between 1.64 and $2.27 \mathrm{~cm}$ [25]. The same research group compared TAUS with TPUS, revealing similar values of displacement in elevation of PFMC, whereas during Valsalva more displacement was measured with TAUS.

Transperineal ultrasound (TPUS) measures the displacement of the $\mathrm{BN}$ with the lift component during maximal voluntary PFMC in the supine position between $0.68 \mathrm{~cm}$ and $0.80 \mathrm{~cm} \mathrm{[21]} \mathrm{and} 0.1 \mathrm{~cm}$ during gentle PFMC [39]. In halfsitting positions BN displacement during PFMC was between $0.62 \mathrm{~cm}$ [27] and $0.95 \mathrm{~cm} \mathrm{[34]} \mathrm{in} \mathrm{asymptomatic} \mathrm{women,}$ whereas Reddy et al. [40] quantified $0.66 \mathrm{~cm}$ of BN motion in a mixed population in the standing position.

During the Valsalva manoeuvre displacements typically ranged between $1.85 \mathrm{~cm}$ [41] in the supine and $1.5 \mathrm{~cm}$ [42] in the lithotomy position versus 1.23 [41] and $1.5 \mathrm{~cm}$ [40] in the standing position. In the supine position and during coughing the ARA displaced between 0.51 and $1.10 \mathrm{~cm}$ [16], whereas the BN motion ranged from 1.84 to $2.10 \mathrm{~cm}$ [41]. Typically for the standing position during coughing, a $\mathrm{BN}$ range of $1.02 \mathrm{~cm}$ [40] and $1.84 \mathrm{~cm}$ [43] had been measured. Representative of the expression of BN mobility during PFMC by the means of an angle, maximal incursion (BN elevation) of $5.6^{\circ}[30]$ and $15^{\circ}$ [44] had been reported. Similar values of excursion (BN displacement in the dorso-caudal direction) were shown during Valsalva with $-24^{\circ}$ [44] and $-25^{\circ}[30]$.

Magnetic resonance imaging studies that measured muscle displacement directly reported pubo-coccygeus muscle displacement during PFMC in the supine position of $0.16 \mathrm{~cm}$ [45] and $0.43 \mathrm{~cm}$ [46] in continent women. BN movement during voluntary PFMC in the sitting position had been recorded by Bo et al. [15] with displacement of $0.83 \mathrm{~cm}$ in continent versus $1.27 \mathrm{~cm}$ in SUI women. When they assessed the Valsalva manoeuvre, a BN descent of $2.2 \mathrm{~cm}$ had been measured for SUI subjects, which was in accordance with another study group [47]. One study compared two measurement methods and reported ARA cranial (ventral) displacement during PFMC in the lithotomy position of $0.7 \mathrm{~cm}$ $(1.1 \mathrm{~cm})$ with MRI and $0.5 \mathrm{~cm}(1.1 \mathrm{~cm})$ with TPUS.

\section{Comparison of data in continent and incontinent women}

Differences in both the magnitude and the direction of displacement became apparent, although the results are not conclusive. While continent women showed significantly more BN elevation during PFMC in one study [48], others only detect a trend [25] or a non-significant difference [15, 21] compared to incontinent subjects. During Valsalva the comparisons revealed that incontinent women had significantly increased $\mathrm{BN}$ descent compared with continent women $[25,49,50]$, while non-significant differences have also been found $[15,26,51]$. During coughing the findings were consistent in showing significant greater displacement in incontinent women [16, 31, 32, 36, 37, 48, 51]. "The Knack" strategy during coughing showed significant more initial ventral displacement of the urethra in continent women [16].

The magnitude of displacement seems to be age-dependent. Comparisons in regard to age demonstrated more displacement during coughs in older women compared to younger women [52] and a greater range of motion in younger women with a voluntary PFMC [53]. Rizk et al. [54] assessed the differences between ethnic groups, revealing greater $\mathrm{BN}$ descent on straining in nulliparous healthy white women than non-white women, which the authors attribute to anthropometric differences as height and weight. Strauss et al. [23] assessed the influence of parity on displacement, 
Fig. 2 Magnetic resonance images in coronal, mid-sagittal and axial planes a-c during Valsalva manoeuvre and $\mathbf{d}-\mathbf{f}$ during straining manoeuvre. Positions of the right diaphragmatic cupola $(R D C)$, left diaphragmatic cupola $(L D C)$, and PRM as well as anterolateral abdominal muscle thickness and abdominal diameters can be seen. The position of the pelvic floor is indicated by the white arrow. Because of the dynamic character of the investigations no "neutral position" of the lumbar spine and the os sacrum was defined, and changes in their positions during the different manoeuvres have been accepted to be adequate. disc $L 4 / 5$ basis of the intervertebral disc L4/5, $S$ pubic symphysis, $C$ coccyx, $R$ rectum, $P C L$ pubo-coccygeal line, $P R M$ pubo-rectalis muscle, $A M$ anterolateral abdominal muscles $L P$ ano-coccygeal raphe of the iliococcygeus muscle (levator plate) [33]
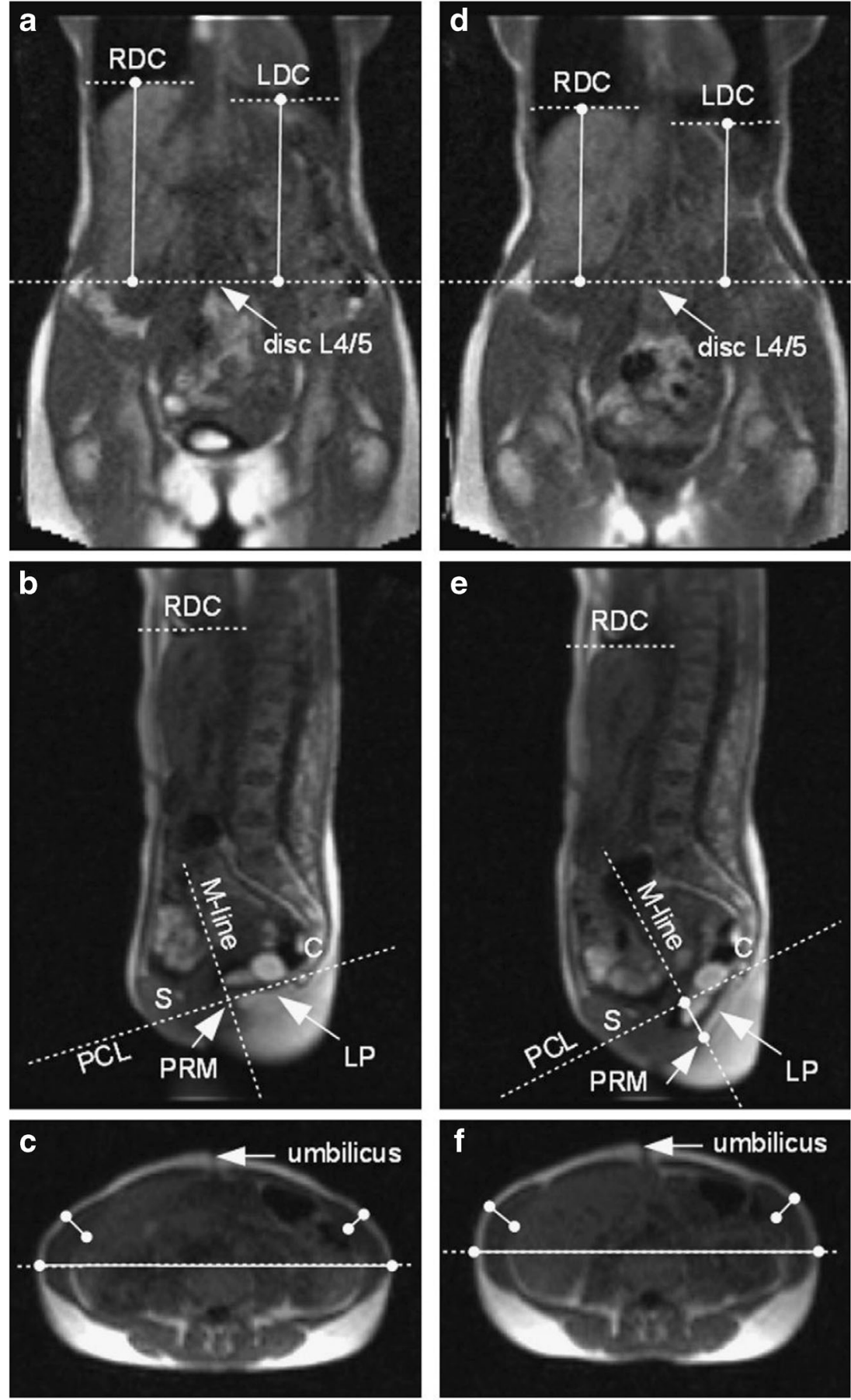

Valsalva maneuver

Straining maneuver demonstrating a significant greater amplitude of organ descent on Valsalva in primiparous compared to nulliparous women and a shift of the force-displacement-vector from ventral to dorsal.

Table 3 displays a summary of the extracted data. For full information (e.g. coordinate system referring to the method of measurement or direction) please see the complete data extraction form, available as electronic supplementary material 2, Table 5).

\section{Discussion}

\section{Main findings}

We found a wide variety of measurement methods, including MRI, several forms of ultrasound, and a broad spectrum of displacement calculations (coordinate systems, motion tracking) for voluntary (squeezing) and reflex tasks (e.g. coughing 


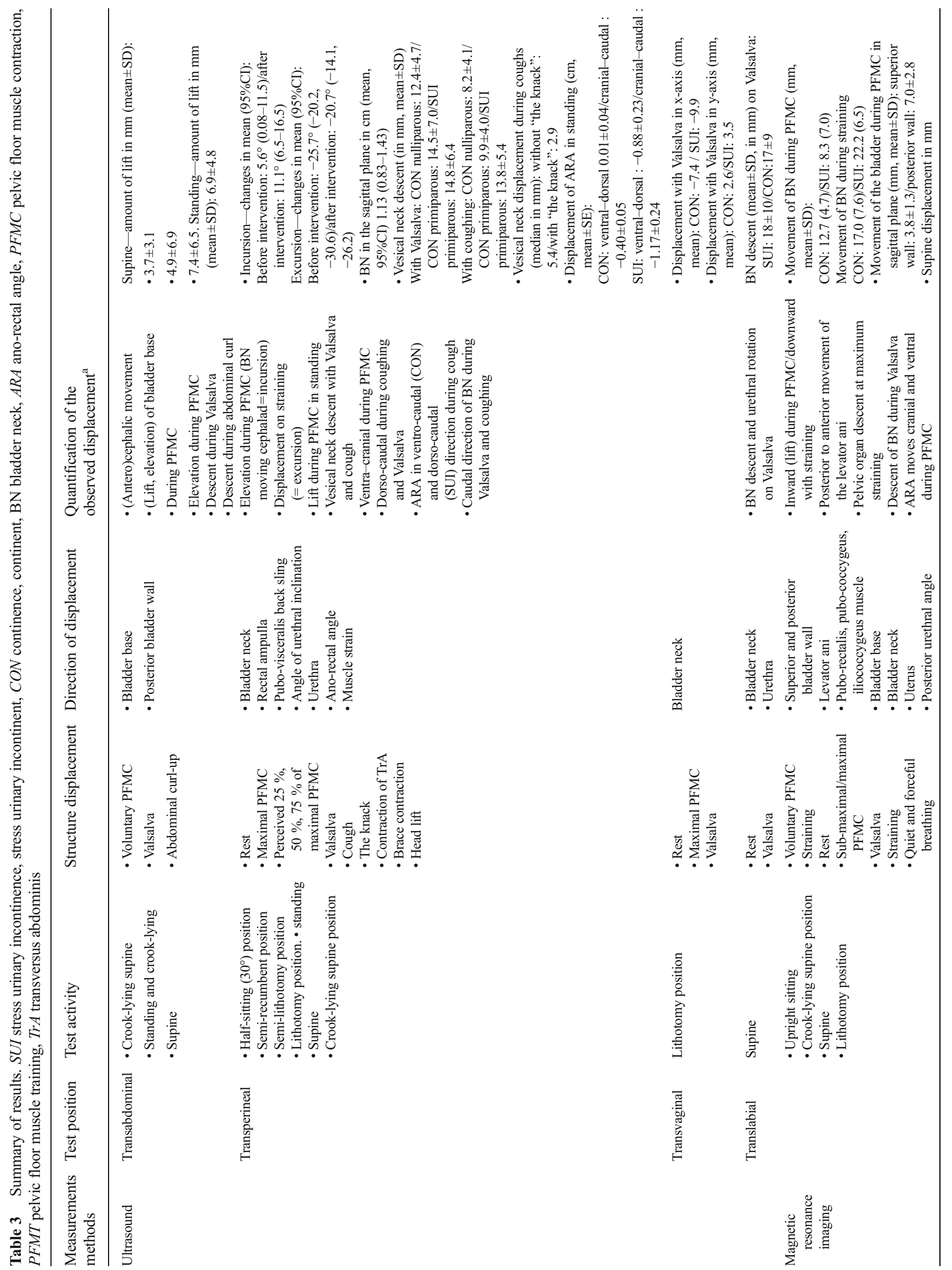


and straining) in different test positions of subjects. The anterior cranial elevating character of voluntary PFM contraction [55], as well as the descent of muscle and organs during impact loads, has been confirmed. Continent women seem to display greater displacement during elevation, while incontinent women perform more downward displacement during coughing and Valsalva manoeuvres.

The collected data also review the major limitations of the methods used. The ultrasound approach cannot visualise the PFM directly. Movements of the bladder base, BN or ARA are surrogate markers for PFM activity. PFMC will elevate the ano-rectal region, and as an indirect effect, other pelvic organs are displaced cranially. Many authors critically evaluate TAUS as lacking a bony landmark $[25,38,56]$. Comparing TAUS with TPUS showed $85 \%$ agreement for assessing the direction of movement during voluntary contraction [57]. Other authors addressed potential transducer movement with TAUS leading to misinterpretation [58]. Reddy et al. [40] took the probe movement into account when assessing the magnitude of BN movement by TPUS and thereby suggested correcting the value of BN motion. Peng et al. [35] tried to correct probe movement by the use of a six-degrees-offreedom measuring device.

Magnetic resonance imaging methods are limited by slow acquisition speed to identify PFM responses to stress-inducing forces [53]. Early MRI took $1.5 \mathrm{~min}$ [59] per image, thus only allowing voluntary contractions to be assessed. In 2010, a duration of $10 \mathrm{~s}$ for sustained PFMC was reported [46]. Constantinou et al. [53] suggest the use of a fast MRI system to visualise the guarding reflex. Ultrasound image processing by motion tracking has been established as a useful tool to measure PFM during fast and stressful events (e.g. coughing) $[35,36,60]$.

The Valsalva manoeuvre, i.e. a forced expiration against a closed glottis and contracted diaphragm and abdominal wall, is routinely used in urogynaecological diagnostics to assess $\mathrm{BN}$ mobility. While the levator muscle co-activation is probably a physiological reaction to maintain continence, it is regarded as a confounder that prevents organ descent [55, 61]. Usually, the Valsalva manoeuvre is instructed as a "strong push" or "bearing down" effort and many authors use "straining" synonymously. However, it has been demonstrated that those tasks show differential effects on PFM with elevation during Valsalva and descent during straining [32]. In a study by Thompson et al. [62] Valsalva was associated with an increase in PFM activation. Pregazzi et al. [48] showed that the urethral beta angle significantly lowered with straining in incontinent women, whereas it increased in continent women. In contrast to common practice a Valsalva manoeuvre seems to protect the contracted and elevated PF against high intra-abdominal pressure and therefore is not so appropriate for provoking pelvic organ descent. It should not be equated to the straining manoeuvre, which physiologically 
rather leads to PFM relaxation and PF descent. As it has become evident that Valsalva and straining are not the same manoeuvres, attempts to standardise these manoeuvres should be made. Therefore, we suggest the differentiated perspective and specific instruction of Talasz et al. [32], as lacking differentiation between the Valsalva manoeuvre and straining manoeuvre may adversely affect research findings and may negatively influence PFM re-education. They instructed the Valsalva manoeuvre as "take a breath, then close the mouth, pinch the nostrils with the thumb and the index finger, than blow air forcefully toward the blocked mouth and nostrils and direct the increasing pressure into the ears", and the straining manoeuvre as "take a breath, then contract the abdominal muscles and strain downwards with the intention to evacuate stool or urine".

Also, the standardisation of testing positions should be pursued and should also include the posture of the lumbar spine as the degree of lumbar lordosis influences the co-activation between PFM and abdominal muscles [63] and may affect displacement too.

\section{Strengths and limitations}

To the best of our knowledge this is the first systematic review on the topic of PFM displacement and dynamics. The sensitivity of this review, resulting in an important number of studies found, can be viewed as a strength. The multidisciplinary authorship comprising a urogynaecologist, physiotherapists, sports and movement scientists may be another advantage of this study. However, the authors are aware of some limitations in this review. First, since most of the studies included had an observational design, sources of bias cannot be eliminated. The second limitation was the substantial heterogeneity among the findings, which did not allow for a meta-analysis.

\section{Interpretation}

Pelvic floor muscle displacement is influenced by multiple factors, including the task, the diagnostic instrument, the test position, continence status, age, muscle condition and fascial structures.

The magnitude of displacement should be interpreted with caution as a larger displacement does not necessarily represent a more forceful contraction $[64,65]$ and the strength of PFM contraction does not always correlate with continence state [10]. A larger lift may result from either a forceful PFM contraction or increased fascial laxity. In contrast, a small lift observed during a voluntary PFM contraction may indicate either a weak contraction or high PFM resting activity [37, 57, 58]. Kelly et al. [24] found displacement to be positiondependent too, with more displacement of the bladder base in the standing than in the crook-lying position.
Lower urethral displacement in asymptomatic women during a cough is attributed to a stiffer pelvic floor that supports the urethra [36], whereas urethral hypermobility in SUI patients is attributed to the weakening of urethra supporting structures [50]. This is consistent with "The Knack" strategy, which is the PFM contraction preceding a cough [52]. PFM laxity is more common in SUI women [47] and nulliparas display a greater stiffness during a cough compared with primiparas [51]. A lower magnitude of displacement is also attributed to stronger fascial attachments [36]. An MRI study confirmed fascial anatomical defects in SUI women [22]. Barbic et al. [31] found that greater compliance of BN support with a delay in muscle activation and early onset of PFM activation was interpreted as a pretension of endopelvic fascia and vaginal wall tissues.

Some authors advocate the concept of "muscle strain" $\varepsilon$. Rahmanian et al. [30] calculated the quotient of $\Delta \mathrm{L}$ and L0 ( $\mathrm{L}=$ length) and thus focused on the elastic properties of tissues. Thyer et al. [66] viewed muscle strain $\varepsilon$ as a surrogate for "elasticity" of PFM. In a similar approach Howard et al. [51] performed a stiffness calculation by dividing the pressure exerted during a particular effort by the urethral descent $\left(\mathrm{cmH}_{2} \mathrm{O} / \mathrm{mm}\right)$. Conversely, Barbic et al. [31] defined the "compliance C" of supporting structures as an inverted evaluation to "stiffness", calculating $\mathrm{C}$ as a quotient between $\mathrm{BN}$ mobility and the rectal pressure during coughing.

\section{Conclusion}

Questions on the specific muscle action of PFM remain unanswered. Komi declared that concentric and eccentric muscle actions in the pure form are seldom found and suggested that the natural muscle function might be more often a stretch and shortening cycle (SSC) [14]. However, knowledge about this combination of eccentric and concentric actions in the PFM during impact load is still inadequate. Impact or any rise in intra-abdominal pressure that provokes a caudal/posterior movement may be interpreted as an eccentric action. However, it is still unknown as to what extent the contraction during high-impact exercises represents a SSC. Further investigations that combine displacement imaging with EMG could assess the activation patterns of the PFM during various manoeuvres and between diverse cohorts.

The development of new diagnostic approaches is needed, as the acquisition times of the present imaging procedures are too slow and imaging cannot be carried out during running or jumping activities.

Stiffness is a concept that is inevitably associated with PFM biomechanics. The importance and role of fascial structures that are attached to the PFM are not yet fully appreciated in investigations of PFM displacement. 
Owing to the heterogeneity of the studies evaluated and the study results, this systematic review emphasises the need for a standardised terminology of PFM function and dysfunction as recommended by the Pelvic Floor Clinical Assessment Group of the International Continence Society [67]. Similarly, as, for example, the differentiation between Valsalva and straining manoeuvres revealed, a standardised performance of tasks should be attempted.

The body of research on PFM displacement summarised the knowledge of SUI pathophysiology. Nevertheless, there is a lack of comprehension of the SUI pathomechanism of involuntary, reflexive activation during functional activities. We therefore propose that future investigations should focus on involuntary reflexive contractions during functional activities (e.g. running, jumping) to elucidate PFM dynamics and muscle action during fast and stressful impact manoeuvres.

Acknowledgements We would like to thank Jacqueline Bürki for proofreading the article.

\section{Conflicts of interest None.}

Authors' contributions LM conceived and designed the review, performed the literature search, collected studies and drafted the article. $\mathrm{MH}$, as the second reviewer, supported the screening and data extraction process. TJ contributed to the design of the study, confirmed the methodological assessment and customised the data extraction form. KA contributed with additional literature and supported the draft. RL advised in cases of disagreement between the two reviewers and supported the final draft. All authors edited the article and approved its publication.

Funding None.

\section{References}

1. Monz B, Pons ME, Hampel C, Hunskaar S, Quail D, Samsioe G, Sykes D, Wagg A, Papanicolaou S (2005) Patient-reported impact of urinary incontinence - results from treatment seeking women in 14 European countries. Maturitas 52 [Suppl 2]:S24-S34. doi:10. 1016/j.maturitas.2005.09.005

2. Krause C, Wells T, Hughes S, Brink C, Mayer R (2003) Incontinence in women: effect of expectancy to regain control and severity of symptoms on treatment outcomes. Urol Nurs 23(1):54

3. Papanicolaou S, Hunskaar S, Lose G, Sykes D (2005) Assessment of bothersomeness and impact on quality of life of urinary incontinence in women in France, Germany, Spain and the UK. BJU Int 96(6):831-838

4. Bo K (2004) Urinary incontinence, pelvic floor dysfunction, exercise and sport. Sports Med (Auckland, NZ) 34(7):451-464

5. Nygaard I, DeLancey JO, Arnsdorf L, Murphy E (1990) Exercise and incontinence. Obstet Gynecol 75(5):848-851

6. Luginbuehl H, Baeyens JP, Taeymans J, Maeder IM, Kuhn A, Radlinger L (2014) Pelvic floor muscle activation and strength components influencing female urinary continence and stress incontinence: A systematic review. Neurourol Urodyn doi: 10.1002/ nau. 22612

7. Verelst M, Leivseth G (2007) Force and stiffness of the pelvic floor as function of muscle length: a comparison between women with and without stress urinary incontinence. Neurourol Urodyn 26(6): 852-857

8. Shishido K, Peng Q, Jones R, Omata S, Constantinou CE (2008) Influence of pelvic floor muscle contraction on the profile of vaginal closure pressure in continent and stress urinary incontinent women. J Urol 179(5):1917-1922. doi:10.1016/j.juro.2008.01.020

9. Deffieux X, Hubeaux K, Porcher R, Ismael SS, Raibaut P, Amarenco G (2008) Abnormal pelvic response to cough in women with stress urinary incontinence. Neurourol Urodyn 27(4):291296. doi:10.1002/nau.20506

10. Morin M, Bourbonnais D, Gravel D, Dumoulin C, Lemieux MC (2004) Pelvic floor muscle function in continent and stress urinary incontinent women using dynamometric measurements. Neurourol Urodyn 23(7):668-674. doi:10.1002/nau.20069

11. Morin M, Dumoulin C, Gravel D, Bourbonnais D, Lemieux MC (2007) Reliability of speed of contraction and endurance dynamometric measurements of the pelvic floor musculature in stress incontinent parous women. Neurourol Urodyn 26(3):397-403

12. Constantinou CE (2009) Dynamics of female pelvic floor function using urodynamics, ultrasound and magnetic resonance imaging (MRI). Eur J Obstet Gynecol Reprod Biol 144:S159-S165

13. Komi PV (2003) Strength and power in sport, 2nd edn. Blackwell Scientific Publications, Oxford

14. Komi PV (2000) Stretch-shortening cycle: a powerful model to study normal and fatigued muscle. J Biomech 33(10):1197-1206

15. Bo K, Lilleas F, Talseth T, Hedland H (2001) Dynamic MRI of the pelvic floor muscles in an upright sitting position. Neurourol Urodyn 20(2):167-174

16. Jones LR, Peng Q, Humphrey V, Stokes M, Payne C, (2009) Constantinou C Effect of the knack on the pelvic floor: evaluated by $2 \mathrm{~d}$ real time ultrasound and image processing methods. In: ICS Annual Meeting of the International Continence Society. ICS

17. Raizada V, Bhargava V, Jung SA, Karstens A, Pretorius D, Krysl P, Mittal RK (2010) Dynamic assessment of the vaginal high-pressure zone using high-definition manometery, 3-dimensional ultrasound, and magnetic resonance imaging of the pelvic floor muscles. Am J Obstet Gynecol 203(2):172.e1-172.e8. doi:10.1016/j.ajog.2010. 02.028

18. Sayers A (2008) Tips and tricks in performing a systematic review. Br J Gen Pract 58(547):136

19. Liberati A, Altman DG, Tetzlaff J, Mulrow C, Gøtzsche PC, Ioannidis JP, Clarke M, Devereaux P, Kleijnen J, Moher D (2009) The PRISMA statement for reporting systematic reviews and metaanalyses of studies that evaluate health care interventions: explanation and elaboration. Ann Intern Med 151(4):W-65-W-94

20. Sampson M, McGowan J, Cogo E, Grimshaw J, Moher D, Lefebvre C (2009) An evidence-based practice guideline for the peer review of electronic search strategies. J Clin Epidemiol 62(9):944-952

21. Chen R, Song Y, Jiang L, Hong X, Ye P (2011) The assessment of voluntary pelvic floor muscle contraction by three-dimensional transperineal ultrasonography. Arch Gynecol Obstet 284(4):931936

22. El Sayed RF, El Mashed S, Farag A, Morsy MM, Abdel Azim MS (2008) Pelvic floor dysfunction: assessment with combined analysis of static and dynamic MR imaging findings. Radiology 248(2): $518-530$

23. Strauss C, Lienemann A, Spelsberg F, Bauer M, Jonat W, Strauss A (2012) Biomechanics of the female pelvic floor: a prospective trail of the alteration of force-displacement-vectors in parous and nulliparous women. Arch Gynecol Obstet 285(3):741-747

24. Kelly M, Tan B, Thompson J, Carroll S, Follington M, Arndt A, Seet M (2007) Healthy adults can more easily elevate the pelvic floor in standing than in crook-lying: an experimental study. Aust J Physiother 53(3):187-191 
25. Thompson JA, O’Sullivan PB, Briffa NK, Neumann P (2007) Comparison of transperineal and transabdominal ultrasound in the assessment of voluntary pelvic floor muscle contractions and functional manoeuvres in continent and incontinent women. Int Urogynecol J 18(7):779-786

26. Dietz H, Eldridge A, Grace M, Clarke B (2004) Pelvic organ descent in young nulligravid women. Am J Obstet Gynecol 191(1):95-99

27. Baessler K, Junginger B (2008) Bladder neck elevation with different levels of effort of pelvic floor muscle contraction. In: ICS 2008 Annual Meeting of the International Continence Society (ICS)

28. Schaer GN, Koechli OR, Schuessler B, Haller U (1995) Perineal ultrasound for evaluating the bladder neck in urinary stress incontinence. Obstet Gynecol 85(2):220-224

29. Balmforth JR, Mantle J, Bidmead J, Cardozo L (2006) A prospective observational trial of pelvic floor muscle training for female stress urinary incontinence. BJU Int 98(4):811-817

30. Rahmanian S, Jones R, Peng Q, Constantinou CE (2008) Visualization of biomechanical properties of female pelvic floor function using video motion tracking of ultrasound imaging. Stud Health Technol Inform 132:390-395

31. Barbič M, Kralj B, Cör A (2003) Compliance of the bladder neck supporting structures: importance of activity pattern of levator ani muscle and content of elastic fibers of endopelvic fascia. Neurourol Urodyn 22(4):269-276

32. Talasz H, Kremser C, Kofler M, Kalchschmid E, Lechleitner M, Rudisch A (2012) Proof of concept: differential effects of Valsalva and straining maneuvers on the pelvic floor. Eur J Obstet Gynecol Reprod Biol 164(2):227-233

33. Talasz H, Kremser C, Kofler M, Kalchschmid E, Lechleitner M, Rudisch A (2011) Phase-locked parallel movement of diaphragm and pelvic floor during breathing and coughing - a dynamic MRI investigation in healthy females. Int Urogynecol J 22(1):61-68

34. Wise B, Cutner A, Cardozo L, Abbott D, Burton G (1992) The assessment of bladder neck movement in postpartum women using perineal ultrasonography. Ultrasound Obstet Gynecol 2(2):116-120

35. Peng Q, Jones R, Shishido K, Constantinou CE (2007) Ultrasound evaluation of dynamic responses of female pelvic floor muscles. Ultrasound Med Biol 33(3):342-352

36. Lovegrove Jones RC, Peng Q, Stokes M, Humphrey VF, Payne C, Constantinou CE (2010) Mechanisms of pelvic floor muscle function and the effect on the urethra during a cough. Eur Urol 57(6):1101-1110

37. Chehrehrazi M, Arab AM, Karimi N, Zargham M (2009) Assessment of pelvic floor muscle contraction in stress urinary incontinent women: comparison between transabdominal ultrasound and perineometry. Int Urogynecol J 20(12):1491-1496

38. Sherburn M, Murphy CA, Carroll S, Allen TJ, Galea MP (2005) Investigation of transabdominal real-time ultrasound to visualise the muscles of the pelvic floor. Aust J Physiother 51(3):167-170

39. Junginger B, Baessler K, Sapsford R, Hodges PW (2010) Effect of abdominal and pelvic floor tasks on muscle activity, abdominal pressure and bladder neck. Int Urogynecol J 21(1):69-77

40. Reddy AP, DeLancey JO, Zwica LM, Ashton-Miller JA (2001) Onscreen vector-based ultrasound assessment of vesical neck movement. Am J Obstet Gynecol 185(1):65-70

41. McLean L, Varette K, Gentilcore-Saulnier E, Harvey MA, Baker K, Sauerbrei E (2013) Pelvic floor muscle training in women with stress urinary incontinence causes hypertrophy of the urethral sphincters and reduces bladder neck mobility during coughing. Neurourol Urodyn 32(8):1096-1102. doi:10.1002/nau.22343

42. Peschers U, Gingelmaier A, Jundt K, Leib B, Dimpfl T (2001) Evaluation of pelvic floor muscle strength using four different techniques. Int Urogynecol J 12(1):27-30

43. McLean L, Gentilcore-Saulnier E, Baker K, Harvey M-A, Sauerbrei E (2011) Pelvic floor muscle training reduces bladder neck mobility during coughing in women with stress urinary incontinence. In: 2011 International World Physical Therapy Congress 2011

44. Hung H-C, Hsiao S-M, Chih S-Y, Lin H-H, Tsauo J-Y (2011) Effect of pelvic-floor muscle strengthening on bladder neck mobility: a clinical trial. Phys Ther 91(7):1030-1038

45. Da Roza T, Mascarenhas T, De Araujo M, Parente M, Duarte S, Loureiro J, Natal Jorge R (2011) Displacement of the levator ani muscle in continent and incontinent female athlete. In: ICS 2011 Annual Meeting of the International Continence Society (ICS)

46. Delmas V, Ami O, Iba-Zizen M-T (2010) Examen dynamique du muscle élévateur de l'anus de la femme par modélisation vectorielle 3D IRM: étude préliminaire. Bull Acad Natl Med 194(6):969-980

47. Fielding JR, Griffiths D, Versi E, Mulkern R, Lee M, Jolesz F (1998) MR imaging of pelvic floor continence mechanisms in the supine and sitting positions. AJR Am J Roentgenol 171(6):1607-1610

48. Pregazzi R, Sartore A, Bortoli P, Grimaldi E, Troiano L, Guaschino S (2002) Perineal ultrasound evaluation of urethral angle and bladder neck mobility in women with stress urinary incontinence. BJOG: Int J Obstet Gynaecol 109(7):821-827

49. Hol M, Bolhuis CV, Vierhout M (1995) Vaginal ultrasound studies of bladder neck mobility. BJOG: Int J Obstet Gynaecol 102(1):47-53

50. Hu L, Song Y (2009) Three-dimensional Virtual-reality reconstruction of pelvic floor structure for patients with female stress Urinary incontinence. In: ICS 2009 Annual Meeting of the International Continence Society (ICS)

51. Howard D, Miller JM, Delancey JO, Ashton-Miller JA (2000) Differential effects of cough, Valsalva, and continence status on vesical neck movement. Obstet Gynecol 95(4):535

52. Miller JM, Perucchini D, Carchidi LT, DeLancey JO, Ashton-Miller J (2001) Pelvic floor muscle contraction during a cough and decreased vesical neck mobility. Obstet Gynecol 97(2):255-260

53. Constantinou C, Hvistendahl G, Ryhammer A, Nagel LL, Djurhuus J (2002) Determining the displacement of the pelvic floor and pelvic organs during voluntary contractions using magnetic resonance imaging in younger and older women. BJU Int 90(4):408-414

54. Rizk DE, Czechowski J, Ekelund L (2004) Dynamic assessment of pelvic floor and bony pelvis morphologic condition with the use of magnetic resonance imaging in a multiethnic, nulliparous, and healthy female population. Am J Obstet Gynecol 191(1):83-89. doi:10.1016/j.ajog.2003.12.041

55. Dietz HP (2011) Pelvic floor ultrasound in incontinence: what's in it for the surgeon? Int Urogynecol J 22(9):1085-1097

56. Arab AM, Behbahani RB, Lorestani L, Azari A (2009) Correlation of digital palpation and transabdominal ultrasound for assessment of pelvic floor muscle contraction. J Man Manip Ther 17(3):75E$79 \mathrm{E}$

57. Thompson JA, O'Sullivan PB, Briffa K, Neumann P (2005) Assessment of pelvic floor movement using transabdominal and transperineal ultrasound. Int Urogynecol J 16(4):285-292

58. Whittaker JL, Thompson JA, Teyhen DS, Hodges P (2007) Rehabilitative ultrasound imaging of pelvic floor muscle function. J Orthop Sports Phys Ther 37(8):487-498

59. Christensen L, Djurhuus J, Constantinou C (1995) Imaging of pelvic floor contractions using MRI. Neurourol Urodyn 14(3):209 216

60. Peng Q, Jones RC, Constantinou CE (2006) 2D Ultrasound image processing in identifying responses of urogenital structures to pelvic floor muscle activity. Ann Biomed Eng 34(3):477-493

61. Örnö A-K, Dietz H (2007) Levator co-activation is a significant confounder of pelvic organ descent on Valsalva maneuver. Ultrasound Obstet Gynecol 30(3):346-350

62. Thompson JA, O'Sullivan PB, Briffa NK, Neumann P (2006) Differences in muscle activation patterns during pelvic floor muscle contraction and Valsalva maneuver. Neurourol Urodyn 25(2):148155 
63. Sapsford R, Hodges P, Richardson C, Cooper D, Markwell S, Jull G (2001) Co-activation of the abdominal and pelvic floor muscles during voluntary exercises. Neurourol Urodyn 20(1):31-42

64. Bo K, Sherburn M (2005) Evaluation of female pelvic-floor muscle function and strength. Phys Ther 85(3):269-282

65. Yang J-M, Yang S-H, Yang S-Y, Yang E, Huang W-C (2009) Reliability of real-time ultrasound to detect pelvic floor muscle contraction in urinary incontinent women. J Urol 182(5):2392-2396
66. Thyer I, Shek C, Dietz H (2008) New imaging method for assessing pelvic floor biomechanics. Ultrasound Obstet Gynecol 31(2):201205

67. Messelink B, Benson T, Berghmans B, Bo K, Corcos J, Fowler C, Laycock J, Lim P, van Lunsen R, Nijeholt G (2005) Standardization of terminology of pelvic floor muscle function and dysfunction: report from the pelvic floor clinical assessment group of the International Continence Society. Neurourol Urodyn 24(4):374 